\title{
ON THE MARGINAL VALUE OF AN ANTAGONISTIC GAME
}

\author{
Joachim Hartung \\ Institut für Angewandte Mathematik \\ D-53 Bonn, Wegelerstraße 6
}

Let $X$ and $Y$ be the sets of strategies of two antagonistic players, and for $t \in[0, T] \in \mathbb{R}, T>0$,

$$
f(x, y, t): x \times y+\mathbb{R}
$$

may be a payoff function in the parametric two-person zero-sum game

$$
G_{t}:=(X, Y, f(X, Y, t)), t \in[0, T] \text {, }
$$

where it is to maximize over $X$ and to minimize over $Y$.

Definition 1: If $v(t)=\operatorname{val}\left(G_{t}\right)$ is the value of $G_{t}, t \in[0, T]$, then the marginal value of the family of games $\left\{G_{t}\right\} t \varepsilon[0, T]$ in the point $t=0$ is defined as

$$
v_{+}^{\prime}(0):=\lim _{t \rightarrow+0} \frac{v(t)-v(0)}{t}
$$

We consider conditions - different from that given for instance in [3], [5] - for the existence of the marginal value, which enable us to derive from the marginal value theorem a method for solving a wide class of constrained games.

Let $X, Y$ be not empty closed convex subsets of real topological linear spaces.

Definition 2: A function $g: X \times Y \rightarrow \mathbb{R}$ is 'sup-inf compact', if the level sets $\{x \in X \mid g(x, y) \geqslant a\}, y \varepsilon Y, a \varepsilon \mathbb{R}$, and $\{y \varepsilon Y \mid g(x, y) \leqslant b\}, x \varepsilon x$, b $\varepsilon \mathbb{R}$, are compact.

Let $X(t), Y(t)$ denote the sets of optimal strategies in $G_{t}, t \varepsilon[0, T]$.

Theorem 1: (marginal value theorem)

If (i) $f(x, y, t)$ is concave (convex) in $x$ (in $y$ ) for $t \varepsilon[0, T]$,

(ii) $f(x, y, 0)$ is upper (lower) semicontinuous in $x$ (in $y$ ),

(iii) $f(x, y, 0)$ has a saddle point on $X \times Y$,

(iv) $f^{\prime}(x, y, 0):=\left.\frac{\partial f(x, y, t)}{\partial t}\right|_{t=+0}$ exists, and

$O(1, x) \leqslant \frac{f(x, y, t)-f(x, y, 0)}{t}-f^{\prime}(x, y, 0) \leqslant O(1, y)$, for $t \varepsilon(0, T]$, where $O(1, x)$ is independent of $y$ and $O(1, x)+O$ for $t \rightarrow+0$ and 
fixed $x \in X, O(1, y)$ is independent of $x$ and $O(1, y) \rightarrow O$ for $t \rightarrow+0$ and fixed $y \in Y$,

(v) $f(x, y, 0)$ and $f(x, y, t)$ for $t \varepsilon(0, T]$ are sup-inf compact on $X \times Y$,

then $v_{+}^{\prime}(0)$ exists and

$$
\begin{aligned}
v_{+}^{\prime}(0) & =\max _{x \in X(0)} \min _{y \in Y(0)} f^{\prime}(x, y, 0) \\
& =\min _{y \in Y(0)} \max _{x \in X(0)} f^{\prime}(x, y, 0)
\end{aligned}
$$

\section{Proof:}

Because of (i), (iii) and ( $v$ ) the sets $X(t), Y(t)$ are not empty and there exist the values $v(t)$, for $t \in[0, T]$. Let $x_{t} \varepsilon X(t), y_{t} \varepsilon Y(t)$, $t \varepsilon[0, T]$, then we have

$$
\begin{aligned}
E\left(x_{0}, y_{t}, t\right)-E\left(x_{0}, y_{t}, 0\right) & \leqslant v(t)-v(0) \\
& \leqslant f\left(x_{t}, y_{0}, t\right)-f\left(x_{t}, y_{0}, 0\right) .
\end{aligned}
$$

For $t \in\{0, T]$ we get from (iv)

$$
\begin{aligned}
\wedge \wedge_{x \in X} O(t, x)+t f^{\prime}(x, y, 0) & \leqslant f(x, y, t)-f(x, y, 0) \\
& \leqslant o(t, y)+t \cdot f^{\prime}(x, y, 0)
\end{aligned}
$$

and with (2)

$$
\begin{aligned}
t \cdot f^{\prime}\left(x_{0}, y_{t}, 0\right)+o\left(t, x_{0}\right) & \leqslant f\left(x_{0}, y_{t}, t\right)-f\left(x_{0}, y_{t}, 0\right) \\
& \leqslant v(t)-v(0) \\
& \leqslant f\left(x_{t}, y_{0}, t\right)-f\left(x_{t}, y_{0}, 0\right) \\
& \leqslant t \cdot f^{\prime}\left(x_{t}, y_{0}, 0\right)+o\left(t, y_{0}\right) .
\end{aligned}
$$

From (v) we get

$$
\begin{aligned}
& f^{\prime}(x, y, 0) \geqslant \min _{y \in Y} f^{\prime}(x, y, 0)=f^{\prime}(x, y(x), 0)>-\infty \\
& \text { and } \\
& f^{\prime}(x, y, 0) \leqslant \max _{x \in X} f^{\prime}(x, y, 0)=: f^{\prime}(x(y), y, 0)<+\infty,
\end{aligned}
$$

such that with (4):

$$
\begin{aligned}
t \cdot f^{\prime}\left(x_{0}, y\left(x_{0},, 0\right)+o\left(t, x_{0}\right)\right. & \leqslant v(t)-v(0) \\
& \leqslant t \cdot f^{\prime}\left(x\left(y_{0}\right), y_{0}, 0\right)+o\left(t, y_{0}\right),
\end{aligned}
$$

which means that

$$
\lim _{t \rightarrow+0} v(t)=v(0)
$$

Diviaing in (4) by $t,(5)$ yields 
(8)

$$
\begin{aligned}
& f^{\prime}\left(x_{0}, y_{t}, 0\right)+o\left(1, x_{0}\right) \leqslant f^{\prime}\left(x\left(y_{0}\right), y_{0}, 0\right)+o\left(1, y_{0}\right), \text { and } \\
& f^{\prime}\left(x_{t}, y_{0}, 0\right)+o\left(1, y_{0}\right) \geqslant f^{\prime}\left(x_{0}, y\left(x_{0}\right), 0\right)+o\left(1, x_{0}\right) \text {, }
\end{aligned}
$$

which by $(v)$ means, that the $x_{t}, y_{t}$ are elements of compact sets independent of $t$. Therefore $\left\{x_{t}\right\}_{t \rightarrow+0},\left\{y_{t}\right\}_{t \rightarrow+0}$ have accumulation points $\hat{\mathrm{x}} \varepsilon \mathrm{X}, \hat{\mathrm{Y}} \in \mathrm{Y}$ and convergent subsequences $\left\{\mathrm{x}_{\mathrm{t}_{\mathrm{n}}}\right\}_{\mathrm{n} \in \mathbb{N}} \subset\left\{\mathrm{x}_{\mathrm{t}}\right\}_{t \rightarrow+0}$, $\left\{y_{n}\right\}_{n \in \mathbb{N}} C\left\{y_{t}\right\}_{t \rightarrow+0}$, such that

$$
\begin{aligned}
& \lim _{n \rightarrow \infty} x_{t_{n}}=\hat{x}, \\
& \lim _{n \rightarrow \infty} y_{t_{n}}=\hat{y} .
\end{aligned}
$$

By (ii) we get for all $x \in X$

$$
f(x, \hat{y}, 0) \leqslant \frac{1 i m}{t_{n} \rightarrow+0} f\left(x, y t_{n}, 0\right) \leqslant \overline{l i m}_{t_{n} \rightarrow+0} f\left(x, y t_{n}, 0\right)
$$

and with (iv) and (5)

$$
\begin{aligned}
& v\left(t_{n}\right)-f\left(x, y_{t_{n}}, 0\right) \geqslant f\left(x, y_{t_{n}}\right)-f\left(x, y_{t_{n}}, 0\right) \\
& \geqslant t_{n} \cdot f^{\prime}(x, y(x), 0)+o\left(t_{n}, x\right), \\
& \frac{t_{n}^{1 i m}}{++0}\left(v\left(t_{n}\right)-f\left(x, y_{t_{n}}, 0\right)\right) \geqslant 0,
\end{aligned}
$$

and by (7)

$$
\overline{\lim }_{t_{n} \rightarrow+0} f\left(x, y_{t_{n}}, 0\right) \leqslant v(0)
$$

Because of (9) this gives

$$
f(x, \hat{y}, 0) \leqslant v(0) \text { for all } x \in X \text {, }
$$

i.e. $\hat{y}$ is an optimal strategy in $G_{0}$. Analogously you show $\hat{x} \varepsilon X(0)$. From inequality (4) it follows

$$
\begin{aligned}
f^{\prime}\left(x_{0}, y_{t}, 0\right)+0\left(1, x_{0}\right) \leqslant & \frac{v(t)-v(0)}{t} \\
\leqslant & f^{\prime}\left(x_{t}, y_{0}, 0\right)+0\left(1, y_{0}\right), \\
& \text { for all } x_{0} \in X(0), y_{0} \varepsilon Y(0),
\end{aligned}
$$

such that

$$
\begin{aligned}
\sup _{x \in X(0)} f^{\prime}\left(x, y_{t^{\prime}}, 0\right)+o(1, x) & \leqslant \frac{v(t)-v(0)}{t} \\
& \leqslant \inf _{y \in Y(0)} f^{\prime}\left(x_{t}, y, 0\right)+o(1, y) .
\end{aligned}
$$

Since $y+f^{\prime}(x, y, 0)$ is lower semicontinuous, also $y \rightarrow \underset{x \in X(0)}{\sup } f^{\prime}(x, y, 0)$ is lower semicontinuous. Similarly, $x \rightarrow \operatorname{ynf}_{\mathrm{y} \in(0)} \mathrm{f}^{\prime}(\mathrm{x}, \mathrm{y}, 0)$ is upper semi-
continuous. 
Let $P$ and $Q$ be the sets of accumulation points of $\left\{x_{t}\right\}_{t \rightarrow+O}$ and $\left\{y_{t}\right\}_{t \rightarrow+0}$, respectively, and for $p \varepsilon P, q \varepsilon Q$

$$
\left\{x_{t(p)}\right\}_{t(p) \rightarrow+0} \subset\left\{x_{t}\right\}_{t \rightarrow+0},\left\{y_{t(q)}\right\}_{t(q) \rightarrow+0} \subset\left\{y_{t}\right\}_{t \rightarrow+0}
$$

may be convergent subsequences such that

$$
\lim _{t(p) \rightarrow+0} x_{t(p)}=p, \quad \lim _{t(q) \rightarrow+0} x_{t(q)}=q .
$$

From (15) we get now

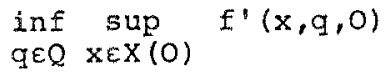

$$
\begin{aligned}
& \leqslant \inf _{q \varepsilon Q} \lim _{(q) \rightarrow+0} \sup _{x \in X(0)} E^{\prime}\left(x, y_{t(q)}, 0\right) \\
& \leqslant \frac{\lim }{t \rightarrow+0} \sup _{x \in X(0)} E^{\prime}\left(x, y_{t}, 0\right) \\
& \leqslant \lim _{t \rightarrow+0} \frac{v(t)-v(0)}{t} \\
& \leqslant \overline{\lim }_{t \rightarrow+0} \frac{v(t)-v(0)}{t} \\
& \leqslant \overline{\lim }_{t \rightarrow+0} \inf _{y \in Y(0)} E^{\prime}\left(x_{t}, y, 0\right)
\end{aligned}
$$

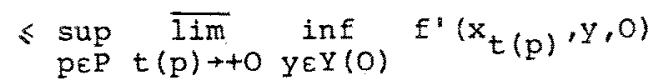

$$
\begin{aligned}
& \leqslant \sup _{p \in P} \inf _{y \in Y(0)} f^{\prime}(p, y, 0)
\end{aligned}
$$

By (13) we have

$$
\mathrm{P} \subset \mathrm{X}(0), \mathrm{Q} \subset \mathrm{Y}(0),
$$

and (16) yields

$$
\begin{aligned}
\inf _{y \in Y(0)} & \sup _{x \in X(0)} f^{\prime}(x, y, 0) \\
\leqslant & \inf _{y \in Q} \sup _{x \in X(0)} f^{\prime}(x, y, 0) \\
\leqslant & \lim _{t \rightarrow+0} \frac{v(t)-v(0)}{t} \\
\leqslant & \prod_{t \rightarrow+0} \frac{v(t)-v(0)}{t} \\
\leqslant & \sup _{x \in P} \inf f^{\prime}(x, y, 0) \\
\leqslant & \sup _{x \in X(0) \inf f_{y}(0)} f^{\prime}(x, y, 0)
\end{aligned}
$$


On the other hand there holds the sup-inf inequality

$$
\sup _{x \in X(0)} \inf _{y \in Y(0)} f^{\prime}(x, y, 0) \leqslant \inf _{y \in Y(0)} \sup _{x \in X(0)} f^{\prime}(x, y, 0) .
$$

Thus $v_{+}^{\prime}(0)=\lim _{t \rightarrow+0} \frac{v(t)-v(0)}{t}$ exists and by the sup-inf compactness of $f^{\prime}(x, y, 0)$ we get

$$
\begin{aligned}
v_{+}^{\prime}(0) & =\max _{x \in X(0)} \min _{y \in Y(0)} f^{\prime}(x, y, 0) \\
& =\min _{y \in Y(0)} \max _{x \in X(0)} f^{\prime}(x, y, 0) .
\end{aligned}
$$

\section{Theorem 2:}

Let $x_{t} \varepsilon X(t), Y(t) \in Y(t), t \varepsilon(0, T]$.

For any accumulation points $\hat{x}, \hat{y}$ of $\left\{x_{t}\right\}_{t \rightarrow+0},\left\{y_{t}\right\}_{t \rightarrow+0}$, respectively, it holds then under the assumptions of Theorem 1:

$$
f^{\prime}(\hat{x}, \hat{y}, 0)=v_{+}^{\prime}(0) \text {. }
$$

\section{Proof:}

Let $x_{t_{n}} \rightarrow \hat{x}$, for $t_{n} \rightarrow+0$, then we get from (15) with the results of Theorem 1:

$$
\begin{aligned}
\sup _{x \in X(0)} f^{\prime}(x, \hat{y}, 0) & \leqslant \underset{t_{n} \rightarrow+0}{\frac{l i m}{\sup _{x \rightarrow X}(0)} f^{\prime}\left(x, y_{t_{n}}, 0\right)} \\
& \leqslant v_{+}^{\prime}(0)=\operatorname{val}\left(x(0), y(0), f^{\prime}(x, y, 0)\right) .
\end{aligned}
$$

Since $\hat{Y} \in Y(O)$ that means, $\hat{Y}$ is an optimal strategy in the game $\left(X(0), Y(0), f^{\prime}(x, y, 0)\right)$. Similarly, $\hat{x}$ is an optimal strategy in this game.

Now we come to an application of the results above to constrained games.

Let $U, V$ be normed real vector spaces, $C \subset U, K \subset V$ not empty closed convex cones, $\mathrm{g}: \mathrm{X} \rightarrow \mathrm{U}, \mathrm{h}: \mathrm{Y} \rightarrow \mathrm{V}$ continuous and concave relative to the cones, and $\phi: X \times Y \rightarrow \mathbb{R}$ be upper-lower semicontinuous, concave-convex and sup-inf compact.

We consider the constrained game

$$
(C G):=(\{x \in X \mid g(x) \in C\},\{y \in Y \mid h(y) \in K\}, \phi(x, y)) \text {. }
$$

With

$$
\begin{aligned}
& d(g(x), C):=\inf _{c \in C}\|g(x)-c\|, \\
& d(h(y), K):=\inf _{k \in K}\|h(y)-k\|,
\end{aligned}
$$

we define for $t \geqslant 0$ 


$$
f(x, y, t):=d(h(y), K)-d(g(x), C)+t \cdot \phi(x, y) .
$$

A solution method for (CG) is given by the following

Theorem 3:

Let $\left\{t_{n}\right\}_{n \in \mathbb{N}}$ be a positive real nullsequence and (CG) may have admissable strategies.

Then (i) the unconstrained games $\left(X, Y, f\left(x, y, t_{n}\right)\right)$ have optimal strategies

$$
x_{t_{n}}, y_{t_{n}}
$$

(ii) $\left\{x_{t_{n}}\right\}_{n \in N},\left\{y_{t_{n}}\right\}_{n \in N}$ have accumulation points $\hat{x}, \hat{y}$ and $\hat{x}, \hat{y}$ are optimal strategies for (CG).

(iii) $\lim _{n \rightarrow \infty}\left\{\phi\left(x_{t_{n}}, y_{t_{n}}\right)+\frac{1}{t_{n}}(d(h(y), k)-d(g(x), c)\}=\phi(\hat{x}, \hat{y})=\operatorname{val}(C G)\right.$

\section{Proof:}

First we show that $d(g(x), c)$ is continuous and convex in $x$.

$d(., C)$ is continuous on $u, g($.$) is continuous on x$, thus $d(g(), C$.$) is$ continuous on $x, g$ is concave on $c$, i.e. for $x_{1}, x_{2} \in x$ and $0 \leqslant \alpha \leqslant 1$ it holds $g\left(\alpha x_{1}+(1-\alpha) x_{2}\right)-\alpha g\left(x_{1}\right)-(1-\alpha) g\left(x_{2}\right) \varepsilon c$. Then for all $c \varepsilon c$ : $\left.g\left(\alpha x_{1}+(1-\alpha) x_{2}\right)-\alpha g\left(x_{1}\right)-(1-\alpha) g x_{2}\right)+c \varepsilon c$, which gives

$$
\begin{aligned}
\| \alpha g\left(x_{1}\right)+ & (1-\alpha) g\left(x_{2}\right)-c \| \\
= & \| g\left(\alpha x_{1}+(1-\alpha) x_{2}\right)-\left(g\left(\alpha x_{1}+(1-\alpha) x_{2}\right)-\right. \\
& -\alpha\left(g\left(x_{1}\right)-(1-\alpha) g\left(x_{2}\right)+c\right) \| \\
\geqslant & \inf \left\|g\left(\alpha x_{1}+(1-\alpha) x_{2}\right)-d\right\| .
\end{aligned}
$$

Theorem 3.4 in [6] states, that $d(., c)$ is convex. Thus we have

$$
\begin{aligned}
d\left(g\left(\alpha x_{1}+(1-\alpha) x_{2}\right), c\right) & \leqslant d\left(\alpha g\left(x_{1}\right)+(1-\alpha) g\left(x_{2}\right), c\right) \\
& \leqslant \alpha d\left(g\left(x_{1}\right), c\right)+(1-\alpha) d\left(g\left(x_{2}\right), c\right) .
\end{aligned}
$$

It is $f(x, y, O)=d(h(y), K)-d(g(x), C)$. We show $x(0)=\{x \in X \mid$ $g(y) \varepsilon C\}, Y(0)=\{y \varepsilon Y \mid h(y) \varepsilon K\}$. Let $x_{0} \varepsilon X(0), y_{0} \varepsilon Y(0)$, then for all $x \in X, Y \in Y$ it is valid

$$
\begin{aligned}
d\left(h\left(y_{0}\right), k\right)-d(g(x), c) & \leqslant d\left(h\left(y_{0}\right), k\right)-d\left(g\left(x_{0}\right), c\right) \\
& \leqslant d(h(y), k)-d\left(g\left(x_{0}\right), c\right) .
\end{aligned}
$$

It follows $d\left(h\left(y_{0}\right), K\right) \leqslant d(h(y), K)$ for all $y \in Y$.

since (CG) should have admissible strategies, there exists a $y \varepsilon Y$ with $h(y) \varepsilon K$. So $d\left(h\left(y_{0}\right), K\right) \leqslant 0$, i.e. $h\left(y_{0}\right) \varepsilon K$. Analogously it can be shown that $g\left(x_{0}\right) \varepsilon C$, under the assumption that there exists an $x \varepsilon X$ with $g(x) \in C$. Now let $\hat{x} \in X, \hat{y} \varepsilon Y$ such that $g(\hat{x}) \varepsilon C, h(\hat{y}) \varepsilon k$. Then $d(g(\hat{y}), C)=d(h(\hat{y}), K)=0$ and for all $x \in X, y \varepsilon Y$ we have 


$$
-d(g(x), c) \leqslant 0 \leqslant d(g(y), K),
$$

i.e. $\hat{x} \in X(0), \hat{y} \varepsilon Y(0)$. Further we have $v(0)=0$. $f(x, y, t)$ is sup-inf compact for $t>0$, because $f^{\prime}(x, y, 0)=\phi(x, y)$ has this property and $f(x, y, 0)$ is bounded from above (below) in $x$ (in $y$ ). From this fact part (i) of the theorem follows.

We have shown that the assumptions of theorem 1. are fulfilled, such that (ii) and (iii) follow from the theorems 1 . and 2, respectively from the proofs.

To solve the problem of finding optimal strategies of the games $(X, Y, f(x, y, t))$, you often have to take algorithms which need for convergency the function $f(, \ldots, t)$ to be strictly concave-convex, as for example the successive approximation method given in [1]. If $f$ does not possess this property, we can do the following:

Let $\psi(x, y)$ be a strictly concave-convex upper-lower semicontinuous real valued function on $\mathrm{X} \times \mathrm{Y}$, which is bounded from above (below) in $\mathrm{x}$ (in $\mathrm{y}$ ) by some $a(y) \in \mathbb{R}(b(x) \in \mathbb{R})$. Then we define

$$
F(x, y, t):=f(x, y, t)+t^{2} \psi(x, y) \text {. }
$$

If the conditions of theorem 1, are fulfilled for $f$, then also for $F$. We show this for the condition (iv):

If $o_{f}(1, x) \leqslant \frac{f(x, y, t)-f(x, y, 0)}{t}-f^{\prime}(x, y, 0) \leqslant o_{f}(1, y), t>0$, then we define $O_{F}(1, x):=O_{f}(1, x)+t \cdot b(x), O_{F}(1, y):=O_{f}(1, y)+t \cdot a(y)$ and get

$$
O_{F}(1, x) \leqslant \frac{f(x, y, t)-f(x, y, 0)}{t}+t \cdot \psi(x, y)-f^{\prime}(x, y, 0) \leqslant O_{F}(1, y) .
$$

Since $F(x, y, 0)=f(x, y, 0), F^{\prime}(x, y, 0)=f^{\prime}(x, y, 0)$ this states 1.(iv) for $F$. Furthermore, let $V(t):=\operatorname{val}(x, y, F(x, y, t))$, then $V(0)=v(0)$ and $v_{+}^{\prime}(0)=v_{+}^{\prime}(0)$. Thus in order to compute $v_{+}^{\prime}(0)$, instead of $(x, y, f(x, y, t))$ we can solve the games $(X, Y, F(x, y, t))$, which have unique solutions (for $t>0$ ).

Under certain conditions it can be shown that the accumulation points of the corresponding optimal strategies for $t \rightarrow+0$ are uniquely determined, such that the whole sequences are converging to optimal strategies of the game $\left(X(0), Y(0), f^{\prime}(x, y, 0)\right)$.

This kind of regularization is particularly interesting for the above given method for solving constrained games. 


\section{References}

[1] DEM'JANOV, V.F., Successive Approximation for Finding Saddle Points, Soviet Math. DokI. 8, 6, 1967.

[2] DUGOŠIJA, $\oplus$. u. J.HARTUNG, Uber Stabilität in Zwei-Personen-Null-Summen-Spielen, Matematica Balkanica (Beograd) 4, 1974.

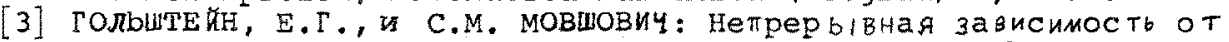
параметра множества решений минимаксной залачи, ЭКономика и матем. мето $46 ! 4,6,1968$.

[4] HARTUNG, J., Penalty-Methoden für Kontrollprobleme und Open-Loop-Differential-Spiele, to appear in: R. Bulirsch, W. Oettli, J. Stoer (eds.) "Optimierungstheorie und optimale Steuerungen", Lect. Not. Math., Springer, Berlin, Heidelberg, New York, 1975.

[5] MILLS, H., Marginal Values of Matrix Games and Linear Programs, in: H.W. Kuhn, A.W. Tucker (eds.) "Linear Inequalities and Related Systems", Princeton University Press, Princeton, New Jersey, 1956.

[6] VAIENTINE, F.A., Convex Sets, McGraw-Hill, New York, 1964. 